Japanese Psychological Research

1967, Vol. 9 , No. $1,35-41$

\title{
THE EFFECT OF SEPARATION AND OVERLAP BETWEEN THE DISK AND THE RING UPON THE CONTOUR EFFECT
}

\author{
TENJI WAKE 1 \\ Shizuoka Tokoha Woman's College
}

\begin{abstract}
The present experiments were concerned with the contour effect. The disk and the ring were provided by a black paper on a white background. They were so arranged as to be a concentric circle. CPT was measured by varying pause between the disk and the ring. Results were as follows: 1. CPT decreases with the increase of width of the ring. 2. The spatial relationships between the disk and the ring are important in the contour effect. 3. There is an optimal overlap between two figures upon CPT. 4. The more separation between them increases, the more CPT decreases.
\end{abstract}

The experiment to be described below is concerned with the so-called contour effect or backward masking. The early work of Werner (1935) on this phenomenon of a disk followed by a ring raised a number of investigations concerning spatial and temporal variables.

An explanation was advanced to account for this phenomenon by Werner. Based on the finding that disappearance of the disk was never observed when the order of two figures was reversed, he assumed that the ring used the partially formed contour of the disk in forming its own contour. Takagi (1927), Sayanagi (1943), and Kolers and Rosner (1960), however, observed the effect of the first figure upon the disk as the second figure. On the other hand, from this assumption, it may be considered that the contour effect is maximal, when the concentric ring is used whose inner border coincides exactly with the border of the disk. But as confirmed by Kolers and Rosner, the disk does not have to be as large as in diameter as the inner circumference of the ring. According to them, at a slight separation between both figures, disappearance of the disk is maxi-

1 The writer would like to thank Prof. Yohei Wada of Tokyo Metropolitan University who gave the various advices in the present study. mal. Such findings cast doubt on Werner's assumption.

Werner's other interpretation is the view that ring-like character with respect to width of the ring is an important factor underlying the contour effect (Werner's impression theory). In our previous work (Wake, 1965b), it was shown that this interpretation seemed to be applicable to the effect of width of the ring. As to the effect of brightness-difference, however, their relative change between the disk and the ring produced the effect on the degree of the contour effect. To provide, from these findings, a satisfactory explanation of this phenomenon, this ring-like character must be accounted for as the relative effect between the disk and the ring.

The aim of the experiments reported here is further to examine the contour effect in view of the findings reported by Werner.

\section{General Method}

Apparatus: As shown in the previous experiments, the tachistoscope (Harvard-type) was used. Three visual fields were presented in succession : a fixation field, a first figure field and a second figure field. Luminance of the fixation field was 174 lux. That of the first 
TABLE 1

Relationship between $\mathcal{T}_{2}$ and inner diameter of the ring (msec)

\begin{tabular}{clcccc}
\hline \multirow{2}{*}{$T_{2}$} & & \multicolumn{4}{c}{ Inner diameter of the ring } \\
\hline \multirow{3}{*}{$25 \mathrm{msec}$} & & $9 \mathrm{~mm}$ & $11 \mathrm{~mm}$ & $13 \mathrm{~mm}$ & $15 \mathrm{~mm}$ \\
\hline & KW & 53.33 & 61.67 & 61.67 & 66.67 \\
& WK & 40.00 & 41.67 & 50.00 & 53.33 \\
& IK & 73.33 & 73.33 & 78.33 & 83.33 \\
& Mean & 55.55 & 58.89 & 63.33 & 67.78 \\
\hline \multirow{3}{*}{$65 \mathrm{msec}$} & KW & 53.33 & 55.00 & 61.67 & 71.67 \\
& WK & 53.33 & 55.00 & 60.00 & 63.33 \\
& IK & 71.67 & 73.33 & 75.00 & 95.00 \\
& Mean & 59.44 & 61.11 & 65.56 & 76.66
\end{tabular}

figure field was 127 lux. That of the second figure field was 150 lux.

Procedure: Let duration of presentation of the first figure be $T_{1}$, that of the second figure be $T_{2}$, and that of pause between both figures be $\Delta t$. The experiment began with the measure of the critical pause threshold (CPT) in succession of two figures. By varying $\Delta t$ progressively, the threshold of $\Delta t$ at which the first figure was seen for the first time was measured under a given $T_{1}$ and $T_{2}$. The psychophysical method employed here was the ascending series of the method of limits, which consist of six trials.

Prior to experiment, three minutes lightadaptation for the fixation field was done. During the light-adaptation, a subject was also asked to report in detail what he observed.

The figures employed were made of an achromatic paper on a white cardboard.

Subjects: Three subjects $(S s)$ were used in the following experiments. The $S_{s}$ were the students of psychology of Tokyo Metropolitan University.

\section{EXPERIMENT 1}

Here the effect of width of the ring upon CPT was examined under the conditions which differed from the previous study.

Conditions: Four disks were employed as the first figure. The diameters of them were varied: 9, 11, 13 and $15 \mathrm{~mm}$ (Visual angle, $0.67,0.83,0.97$ and $\left.1.13^{\circ}\right)$. The rings as the

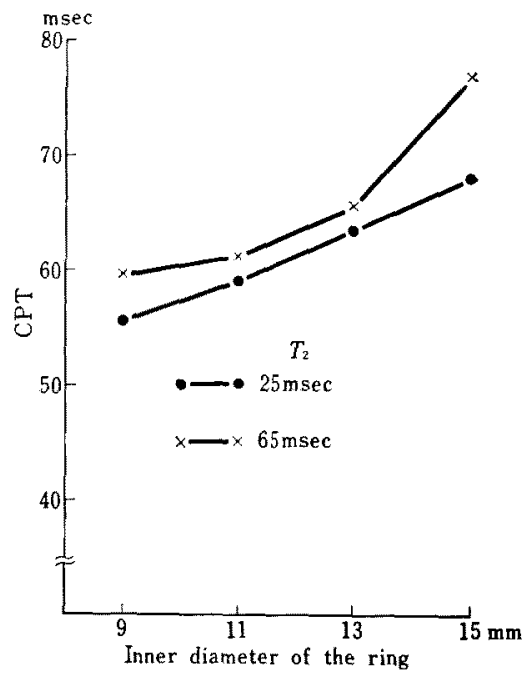

Fig. 1. Average CPTs as a function of inner diameter of the ring.

second figure were always $19 \mathrm{~mm}$ in outer diameter (visual angle, $1.43^{\circ}$ ) and the inner diameters were varied $9,11,13$ and $15 \mathrm{~mm}$, that is, the rings were $5,4,3$ and $2 \mathrm{~mm}$ in width. The border of the disk always coincided exactly with the inner border of the ring. Each of them was 3.28 per cent in reflectance. $T_{1}$ was always $25 \mathrm{msec}$, and $T_{2}, 25$ and $65 \mathrm{msec}$.

Results: The individual CPTs of three $S_{\mathrm{S}}$ are shown in Table 1 . The averages of them are plotted against the inner diameter of the ring in Fig. 1. The curves were fitted to the points visually. As can 
TABLE 2

Relationship between $T_{2}$ and inner diameter of the ring (msec)

\begin{tabular}{|c|c|c|c|c|c|c|c|}
\hline \multirow{2}{*}{$T_{2}$} & \multirow{2}{*}{$S_{\mathrm{s}}$} & \multicolumn{6}{|c|}{ Diameter of the disk } \\
\hline & & & $11 \mathrm{~mm}$ & $13 \mathrm{~mm}$ & $15 \mathrm{~mm}$ & $17 \mathrm{~mm}$ & $19 \mathrm{~mm}$ \\
\hline \multirow{4}{*}{$25 \mathrm{msec}$} & WK & \multirow{4}{*}{ C. } & - & 53.33 & 61.67 & 81.67 & 71.67 \\
\hline & $\mathrm{IK}$ & & - & 78.33 & 83.33 & 115.00 & 115.00 \\
\hline & WD & & - & 53.33 & 81.67 & 105.00 & 93.33 \\
\hline & Mean & & - & 61.66 & 75.56 & 100.56 & 93.33 \\
\hline \multirow{4}{*}{$65 \mathrm{msec}$} & WK & & 55.00 & 85.00 & 85.00 & 90.00 & 80.00 \\
\hline & IK & & 85.00 & 86.67 & 116.67 & 116.67 & 105.00 \\
\hline & WD & & 65.00 & 78.33 & 83.33 & 110.00 & 105.00 \\
\hline & Mean & & 68.33 & 83.33 & 95.00 & 105.56 & 96.67 \\
\hline \multirow{4}{*}{$105 \mathrm{msec}$} & WK & & 68.33 & 73.33 & 78.33 & 81.67 & 78.33 \\
\hline & IK & 0 & 88.33 & 93.33 & 108.33 & 101.67 & 100.00 \\
\hline & WD & & 51.67 & 63.33 & 78.33 & 95.00 & 93.33 \\
\hline & Mean & & 69.44 & 76.67 & 88.33 & 92.78 & 90.55 \\
\hline
\end{tabular}

be seen in Table 1 and Fig. 1, CPT decreases progressively with the increase of width of the ring under each $T_{2}$.

As far as our experimentation is concerned, the diameter of the disk also increases with the increase of the inner diameter of the ring. As shown by many investigations, (Kristoferson, 1957 and Wake 1964), threshold to detect the disk decreased with the increase of its area. In the present experiment, however, CPT expresses the inverse trend. This discrepancy will imply that the contour effect depends upon characteristic of the ring rather than detectability of the disk alone. On the other hand, Okada suggested that the smaller disk was more easily organized than the larger one in this type of the experiment.

A plausible explanation of the result obtained here and the previous study cited, would postulate ring-like character. In terms of this explanation, Okada's suggestion must be further examined under the various conditions.

\section{EXPERIMENT 2}

Here the effect of area of the disk upon
CPT was examined under a fixed ring. Conditions: The ring was $15 \mathrm{~mm}$ in inner diameter and $19 \mathrm{~mm}$ in outer diameter. The diameter of the disks was varied as follows : $11,13,15,17$ (visual angle, $1.27^{\circ}$ ) and $19 \mathrm{~mm}$. $T_{1}$ was always $25 \mathrm{msec}$ and $T_{2}$ was varied as follows: 25, 65 and $105 \mathrm{msec}$. The figures employed were a black paper ( 3.28 per cent).

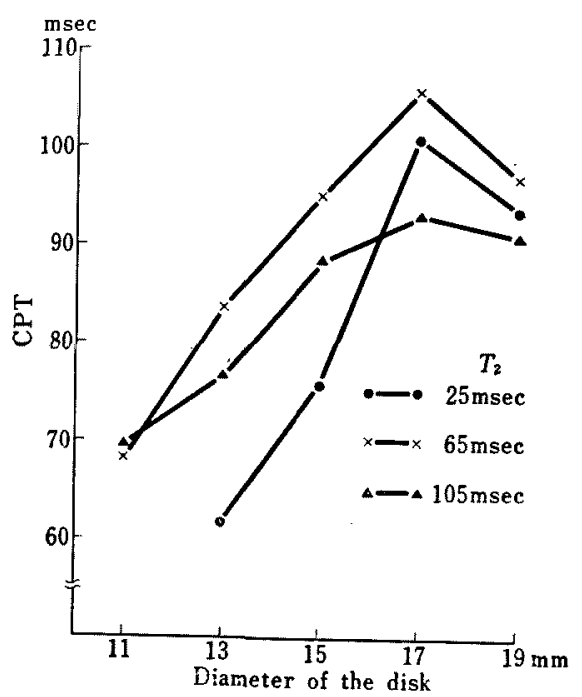

Frg. 2. Average CPTs as a function of diameter of the disk. 
TABLE 3

Relationship between $T_{2}$ and inner diameter of the ring (msec)

\begin{tabular}{rlrrrr}
\hline \multirow{2}{*}{$T_{2}$} & & \multicolumn{4}{c}{ Inner diameter of the ring } \\
\cline { 3 - 6 } & & $9 \mathrm{~mm}$ & $11 \mathrm{~mm}$ & $13 \mathrm{~mm}$ & $15 \mathrm{~mm}$ \\
\hline \multirow{2}{*}{$25 \mathrm{msec}$} & $\mathrm{KW}$ & 103.33 & 105.00 & 80.00 & 68.33 \\
& WK & 101.67 & 86.67 & 70.00 & 65.00 \\
& IK & 141.67 & 150.00 & 120.00 & 90.00 \\
& Mean & 115.56 & 113.89 & 90.00 & 74.44 \\
\hline \multirow{3}{*}{$65 \mathrm{msec}$} & KW & 90.00 & 95.00 & 81.67 & 71.67 \\
& WK & 98.33 & 95.00 & 76.67 & 70.00 \\
& IK & 126.67 & 131.67 & 120.00 & 105.00 \\
& Mean & 105.00 & 107.22 & 92.78 & 82.22 \\
\hline \multirow{3}{*}{$105 \mathrm{msec}$} & KW & 90.00 & 75.00 & 81.67 & 65.00 \\
& WK & 93.33 & 85.00 & 76.67 & 65.00 \\
& IK & 120.00 & 128.33 & 123.33 & 100.00 \\
& Mean & 101.11 & 96.11 & 93.89 & 76.67
\end{tabular}

Results: The individual CPTs of three $S_{\mathrm{s}}$ are shown in Table 2. The averages of them are plotted against the diameter of the disk under each $T_{2}$ in Fig. 2. Table 2 and Fig. 2 suggest that CPT shows a peak at the diameter of $17 \mathrm{~mm}$ of the disk.

This finding should be noted. According to Okada's suggestion, it must be shown that CPT increases with the increase of the diameter of the disk. But the trend obtained here shows a peak at the diameter of $17 \mathrm{~mm}$ of the disk. This trend also disagrees with detectability of the disk in presenting it alone. From those facts, it may be implied that the spatial relationships between the disk and the ring are important in this experimental situation. When the disk was $15 \mathrm{~mm}$ in diameter, the border of the disk coincided exactly with the inner border of the ring. Using the smaller or larger one than this disk, separation or overlap between the disk and the ring appeared. In terms of this view point, data obtained here shows that CPT is maximal at a slight overlap between them.

The effects of separation and overlap upon CPT will need further examination with respect to Werner's assumption.

\section{EXPERIMENT 3}

In the present experiment, it was planned to examine the effect of overlap between two figures upon CPT, being the disk kept

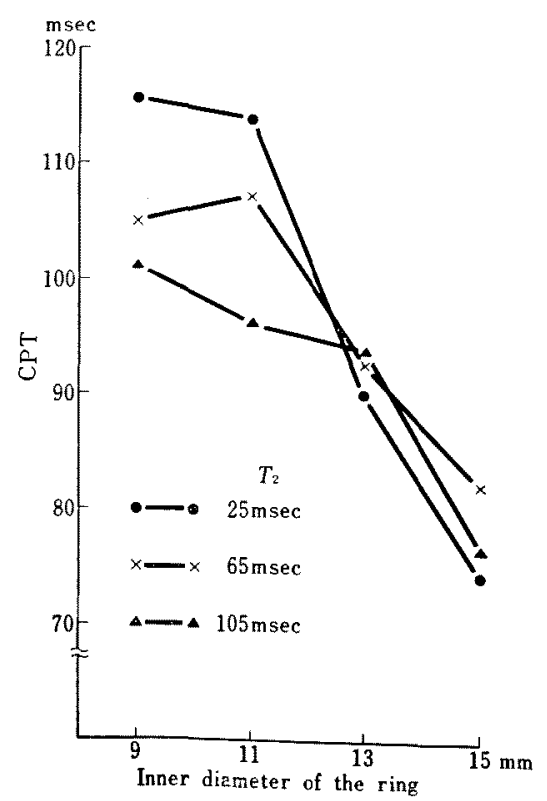

Fro. 3. Average CPTs as a function of inner diameter of the ring. 
TABLE 4

Relationship between $T_{2}$ and inner diameter of the ring (msec)

\begin{tabular}{rlrrrrr}
\hline \multirow{2}{*}{$T_{2}$} & Ss & \multicolumn{5}{c}{ Inner diameter of the ring } \\
\cline { 3 - 7 } & & $15 \mathrm{~mm}$ & $17 \mathrm{~mm}$ & $19 \mathrm{~mm}$ & $21 \mathrm{~mm}$ & $23 \mathrm{~mm}$ \\
\hline \multirow{3}{*}{$65 \mathrm{msec}$} & $\mathrm{KW}$ & 68.33 & 66.67 & 58.33 & 56.67 & 53.33 \\
& WK & 90.00 & 83.33 & 80.00 & 68.33 & 60.00 \\
& IK & 123.33 & 118.33 & 103.33 & 80.00 & 61.67 \\
& Mean & 93.89 & 89.44 & 80.55 & 68.33 & 58.33 \\
\hline \multirow{3}{*}{$105 \mathrm{msec}$} & KW & 58.33 & 65.00 & 55.00 & 46.67 & - \\
& WK & 85.00 & 78.33 & 70.00 & 63.33 & - \\
& IK & 101.67 & 98.33 & 96.67 & 81.67 & - \\
& Mean & 81.67 & 80.55 & 73.89 & 63.89 & -
\end{tabular}

constant.

Conditions: The disk used as the first figure was always $15 \mathrm{~mm}$ in diameter. Four rings were used as the second figure. The outer diameter of them was always $19 \mathrm{~mm}$, and the inner diameter was varied as follows: 9,11 , 13 and $15 \mathrm{~mm}$. Overlaps between the disk and the ring were $3,2,1$ and $0 \mathrm{~mm}$. $T_{1}$ was $25 \mathrm{msec}$ and three levels of $T_{2}, 25,65$ and 105 msec, were employed. Their reflectance was 3.28 per cent.

Results: CPTs of three $S_{\mathrm{s}}$ are shown in Table 3. The averages of them are plotted against the inner diameter of the ring in Fig. 3. As can be seen in Table 3 and Fig. 3, CPT shows a peak at overlap of $2 \mathrm{~mm}$ or $3 \mathrm{~mm}$.

From Werner's assumption, it may be shown that apparent contiguity between two figures is a necessary condition in the contour effect. But as far as our experimentation is concerned, data obtained disagree with his assumption. The trend of $T_{2}$ of 65 msec agrees with that of Experiment 2, which shows a peak at overlap between two figures of $2 \mathrm{~mm}$. From this fact, it will be predicted that the trend of other $\mathcal{T}_{2}$ shows a peak at a slight overlap between them.

\section{EXPERIMENT 4}

In the present experiment, the effect of separation of two figures upon CPT was examined under a fixed disk.

Conditions: The disk as the first figure was $15 \mathrm{~mm}$ in diameter. Area of the ring as the second figure was constant and the inner and outer diameters were varied as follows: (15, $35),(17,36),(19,37),(21,38)$ and $(23 \mathrm{~mm}$, $39 \mathrm{~mm}$ ). Visual angle, 2.63, 2.70, 2.77, 2.83 and $2.90^{\circ}$. Separations between the disk and the ring were $0,1,2,3$ and $4 \mathrm{~mm}$. Their reflectance was 3.28 per cent. $T_{1}$ was $25 \mathrm{msec}$ and $T_{2}$ of 25,65 and $105 \mathrm{msec}$ was used.

Results: Data are shown in Table 4 and Fig. 4. The general tendency is that

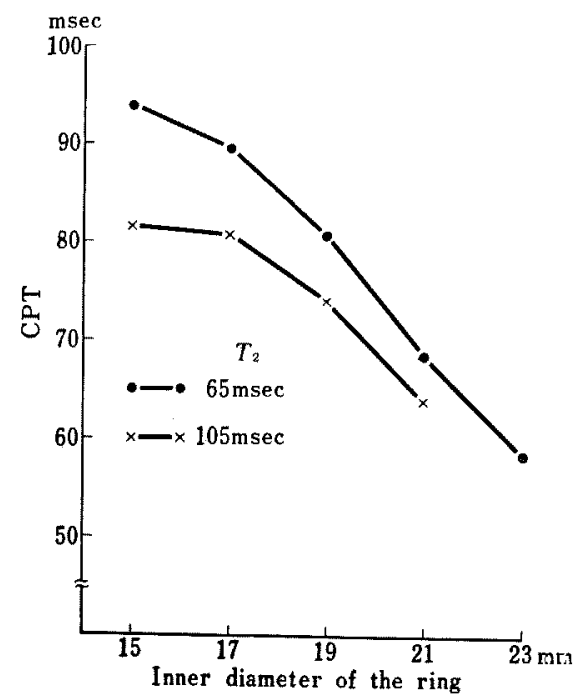

Frg. 4. Average CPTs as a function of inner diameter of the ring. 
CPT decreases with the increase of separation between both figures. In this case, disappearance of the disk can not be obtained at $T_{2}$ of $25 \mathrm{msec}$, which is equal to $T_{1}$.

As mentioned above, although Werner suggested that absolute contiguity of two figures was necessary condition for disappearance of the disk to occur, Kolers and Rosner confirmed that the a mount of masking that a second figure could exert on the first one was inversely related to the amount of visual angle that separated two figures, and was maximal at a slight separation between them. These experiments were done by varying area of the disk or the ring. In Experiment 4, the effect of separation between them was examined under the condition that the ring had a constant area. From the present results, the contour effect occurs even at a slight separation, and disappearance of the disk increases with the decrease of separation between both figures.

\section{Discussion}

With respect to the effect of area of the disk in the disk-ring sequence, Okada suggested that the degree of organization of the disk increased with the decrease of its area. On the other hand, when the disk alone was presented without following the ring, visual threshold of the disk decreased with the increase of area of the disk. If this finding is applied in the disk-ring situation, it may be predicted that disappearance of the disk decreases with the increase of its area.

Under the condition of the absolute contiguity between the disk and the ring, when the diameter of the disk and the inner diameter of the ring were simultaneously varied (the outer diameter of the ring was constant), the variable was area of the disk and width of the ring (Exp. 1). The results show the similar trend as the effect of width of the ring in the previous experiments. This trend does not contradict with the effect of area of the disk as discussed by Okada.

When the disk following the fixed ring was varied in diameter, spatial overlap and separation between both figures appeared (Exp. 2). Under this condition disappearance of the disk is maximal at a slight overlap as shown by Okada. He interpreted his finding in terms of the fact that the contour effect decreased with the decrease of diameter of the disk. As far as the condition in Experiment 2, however, it may be suggested that the results show not only the effect of area of the disk, but also that of separation and overlap between two figures.

In Experiment 3, using the fixed disk, the effect of overlap between both figures was observed when the outer diameter of the ring was constant and its inner diameter was varied. Under this condition, it is predicted that CPT is maximal at a slight overlap between both figures. The effect decreases invariably when overlap between both figures is decreased or increased.

The effect of separation between both figures was observed when the ring had a constant area and the ring was varied in inner and outer diameter (Exp. 4). Disappearance of the disk under this condition increases monotonically as its separation is decreased.

According to Werner's assumption, disappearance of the disk is maximal at absolute contiguity between both figures. Kolers and Rosner showed, however, that disappearance of the disk is maximal at a slight separation between both figures. Although there is such a discrepancy, in order to obliterate the disk, it does not have to be as large in diameter as the inner circumference of the ring. Recently Pollack (1965) found that (1) given an angular separation between the inner and outer figures, a large difference in figure-ground contrast in favor of the masking figure is necessary to produce masking and (2) was lack of parallelism of the inner and outer 
contours inhibited masking. Moreover, the results obtained here and Okada's show that disappearance of the disk increases with the decrease of separation between both figures. These results agree with Werner's assumption. But as to the effect of overlap between two figures, they are not easily reconciled with his assumption. It is evident that these findings show an obvious tendency toward the spatial relationships between two figures. In fact, the disk is not perceived by the effect of the ring alone, but by the relative effect between two figures. Schiller and Smith (1966) also pointed out that the figures employed were always presented in pairs, and under such conditions, $S$ s probably made relative judgements; they reported the absence of the first figure at those pauses where it was less apparent. Such facts imply that Werner's impression theory is necessary to assume spatial interaction occuring between two figures as ring-like character.

There is some possibility that such interactions can explain some of the previous reports on the contour effect (Werner, Okada, Pollack and Wake). More detailed interpretation of these interactions must await further study on the effect of characteristic of two figures, especially must be examined under the view point of the effect upon brightness reduction of the disk.

\section{REFERENCES}

Alpern, M. 1953 Metacontrast. 7. Opt. Soc.
Amer. 43, 648-657.

Fehrer, E. \& RAAB, D. 1962 Reaction time to stimuli masked by metacontrast. 7 . exp. Psychol. 63, 143-147.

Kolers, P. A. \& Rosner, B. S. 1960 On visual masking (metacontrast): Dichoptic observation. Amer. 7. Psychol. 73, 2-21.

Kristofferson, A. B. 1957 Visual detection as infuenced by target form. From discussion as related to military problem. Wulfek, J. W. \& Taylor, J. H. (eds.).

Okada, T. 1937 Studies on contour-effect in successive exposition. Jap. 7. Psychol. 12, (Japanese text 335-359, English summary 3132).

Pollack, R. H. 1965 Effects of figure-ground contrast and contour orientation on figural masking. Psychon. Sci. 2, 369-370.

SAYANAGI, T. 1943 On the influence of the contour figure upon the surrounding ground. Jap. 7. Psychol. 18, 33-44.

Schiller, P. H. \& Smith, M. C. 1966 Detection in Metacontrast. 7. exp. Psychol. 71, 32-39.

TAKAGI, K. 1927 Effect of figure lines on the structure of visual field. Jap. F. Psychol. 2, 217-261.

WaKE, T. 1964 On the effect of form upon visual threshold. Proc. the 28th Annual Meeting of 7 . P. A. Pp. 91. (In Japanese)

WAKE, T. 1965a On the so-called contour effect. Proc. the 29th Annual Meeting of 7. P. A. Pp. 46. (In Japanese)

WAKE, T. 1965b Visual effect of the ring upon critical pause threshold of the disk in disk-ring sequence. 7ap. Psychol. Res. 7, 110-119.

Werner, H. 1935 Studies on contour effect: 1. Qualitative analysis. Amer. 7. Psychol. 47, 40-64.

(Received Feb. 18, 1967) 